\title{
New Class of Metal Bound Molecular Switches Involving $\mathrm{H}$-Tautomerism
}

\author{
Grant J. Simpson, † Simon W. L. Hogan, † Marco Caffio, † Christopher J. Adams, $\neq$ \\ Herbert Früchtl, + Tanja van Mourik, ${ }^{\dagger}$ and Renald Schaub*, ${ }^{\dagger}$
}

†EAStCHEM and School of Chemistry, University of St Andrews, St Andrews, KY16 9ST, United Kingdom

$¥$ \Shool of Chemistry, University of Bristol, Bristol, BS8 1TS, United Kingdom

Corresponding Author $(*): \quad$ renald.schaub@st-andrews.ac.uk.

A potential end-point in the miniaturization of electronic devices lies in the field of molecular electronics, where molecules perform the function of single components. To date, hydrogen tautomerism in unimolecular switches has been restricted to the central macrocycle of porphyrin-type molecules. The present work reveals how $\mathrm{H}$-tautomerism is the mechanism for switching in substituted quinone derivatives, a novel class of molecules with a different chemical structure. We hence reveal that the previous restrictions applying to tautomeric molecular switches bound to a surface are not valid in general. The activation energy of switching in a prototypical quinone derivative is determined using inelastic electron tunneling. Through computational modeling, we show that the mechanism underlying this process is tautomerization of protons belonging to two amino groups. This switching property is retained upon functionalization by the addition of side groups, meaning that the switch can be chemically modified to fit specific applications.

\section{KEYWORDS}

STM, tautomerization, molecular switches, inelastic tunneling

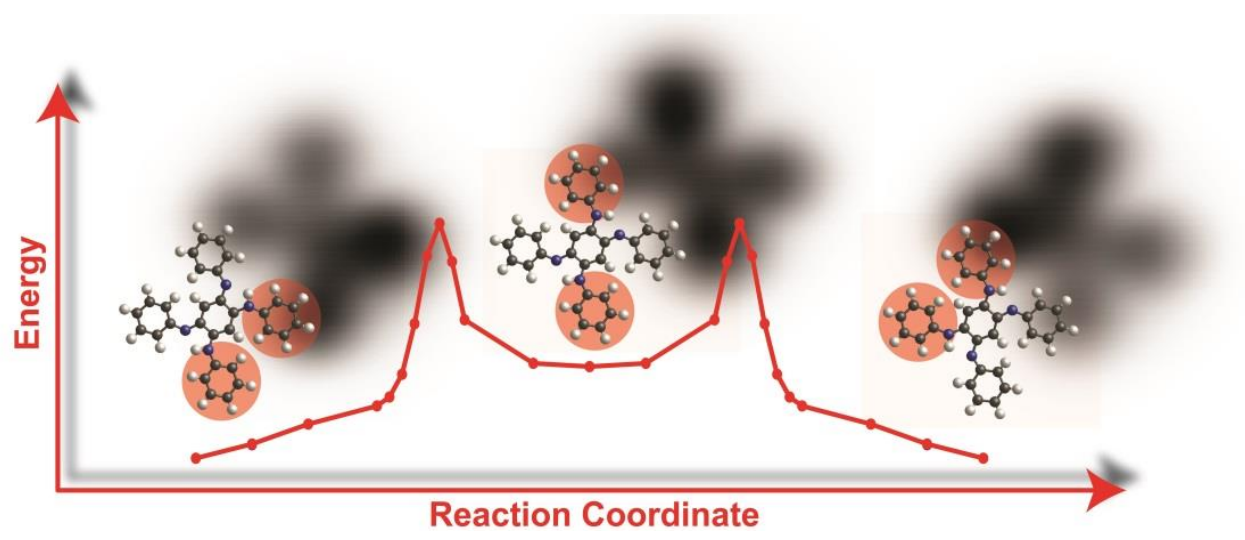

dx.doi.org/10.1021/nl4038517

Received: $\quad$ October 15, 2013

Revised: January 23, 2014

Published: $\quad$ January 28, 2014 
The use of molecules to perform the function of electronic devices such as transistors, ${ }^{1}$ rectifiers, ${ }^{2,3}$ and switches ${ }^{4-6}$ forms the basis of the rapidly expanding field of molecular electronics. Their use in nanoelectronics could provide an ultimate level of miniaturization and efficiency, as well as a possible replacement to semiconductor electronics, which face increased cost and power consumption as their size decreases. Research in this field is still relatively fundamental despite the first publication appearing in 1974,2 due to the difficulties faced in incorporating single molecules into electrical circuits and their functional use at ambient conditions.

Of particular relevance to this letter are molecular switches, which must possess two distinguishable, stable states and have the capacity to switch between these on demand. These basic " 1 " and " 0 " states, which are often discriminated by differences in their conductivities, are vital in the operation of any logical device. Interconversion may be achieved in a number of ways, including interaction with electromagnetic radiation, ${ }^{7}$ presence of an electric field, ${ }^{8}$ application of a mechanical force, ${ }^{4}$ or energy provided by incident electrons. ${ }^{9}$

Progress has also been made in determining the mechanisms behind the observed switching processes. Control of the charge state of a single molecule $^{10}$ and the formation and cleavage of metal-organic bonds in a self-assembled copper complex ${ }^{9}$ constitute two examples. A broad class of molecular switches are those in which the mechanism is a conformational change. These include cis-/trans-isomerization, ${ }^{11,12}$ changes in the position of constituents on a benzene ring, ${ }^{13}$ and, of interest in this study, hydrogen (H)tautomerization. ${ }^{14}$

With regards to molecular switching, $\mathrm{H}$ tautomerization has only been observed in the fixed ring system of porphyrin-type molecules. ${ }^{14}$ In this process, the protons accommodated by the macrocycle switch positions, leading to a change in the HOMO and LUMO, which in turn induces a change in the conductivity across the molecule. A two-level switch was first discovered, involving reversible switching of two protons occupying the cage. ${ }^{14}$ Later, a four-level conductance switch was realized by purposeful removal of one of the protons using the tip of a scanning tunneling microscope (STM). ${ }^{15}$ In this class of molecules, the central macrocycle effectively performs as a protective environment for the active protons. In addition, adsorption on surfaces results in molecular configurations that maintain the two-dimensional macrocycle fairly unperturbed. From gas-phase and solution chemistry, $\mathrm{H}$-tautomerism is, however, wellknown to occur in a plethora of molecules. Solely on the basis of the criteria suggested by these studies (unperturbed, 2-dimensional, and protective encasing), hardly any other candidates could be proven to act as a $\mathrm{H}$-tautomeric molecular switch. Figuring out which other molecules will retain this property when adsorbed on a substrate hence remains a major challenge. Indeed, the vast majority of tautomerically active molecules involve threedimensional moieties, which, upon adsorption on a two-dimensional substrate, will likely deform enough to deactivate tautomerism (rendering it energetically unaffordable).

In the current work, we exploit $\mathrm{H}$-tautomerization in a new class of metal bound molecular switches, based on a quinone derivative. The two molecules studied experimentally share a common threedimensional central chemical unit (the active switching moiety), which has an altogether different structure than that of previously reported $\mathrm{H}$ tautomeric switches. Upon examination of their properties by means of STM, we find that an internal two-way switching mechanism operates in both molecules. In combination with density functional theory (DFT) calculations, we show that proton transfer occurs on the periphery of the common central switching unit, unprotected by other chemical groups. This switch is activated by inelastic tunneling from the STM tip in a single-electron process. We further demonstrate that molecular functionalization (during the synthesis of the molecules) preserves the metal-bound $\mathrm{H}$-tautomeric properties, in that the energy barrier for the mechanism remains unaffected, within experimental errors, by incorporation of side groups.

The central functional unit of these molecules is a 3,6-diimino-1,4-diamino-1,4-cyclohexadiene moiety. As shown in Figure 1a, the $R$ group is substituted with a hydrogen atom, a phenyl group, or a tolyl group to produce 3,6-diimino-1,4-cyclohexadiene1,4-diamine (DCD), N, N'-diphenyl-3,6bis(phenylimino)- 1,4- cyclohexadiene- 1,4- diamine azophenine, AP), and N,N'-ditolyl-3,6-bis(tolylimino)1,4-cyclohexadiene-1,4-diamine (azotolyline, AT), respectively. Of these three molecules, we have conducted our experimental investigations on AP and AT. DCD represents the smallest possible molecular switch based on our quinone derivative, and as such, was investigated theoretically together with AP.

$\mathrm{DCD}, \mathrm{AP}$, and AT exist natively as red polycrystalline solids in the para configuration, whereby identical functional groups occupy opposite positions in the 
central 6-membered ring. Similarly, the forms in which identical groups occupy the 1,2 and 1,3 positions are referred to as ortho and meta, respectively. All three molecules are known to act as bidentate ligands in metal complexes via the lone pairs located on the nitrogen atoms. ${ }^{16-18}$ In addition, further studies have shown that in liquid solution and crystalline solid, a double $\mathrm{H}$-transfer reaction occurs for both para-DCD and para-AP, involving the protons occupying the amino groups. ${ }^{19-21}$ It is this observation that reveals their potential use as novel molecular switches.
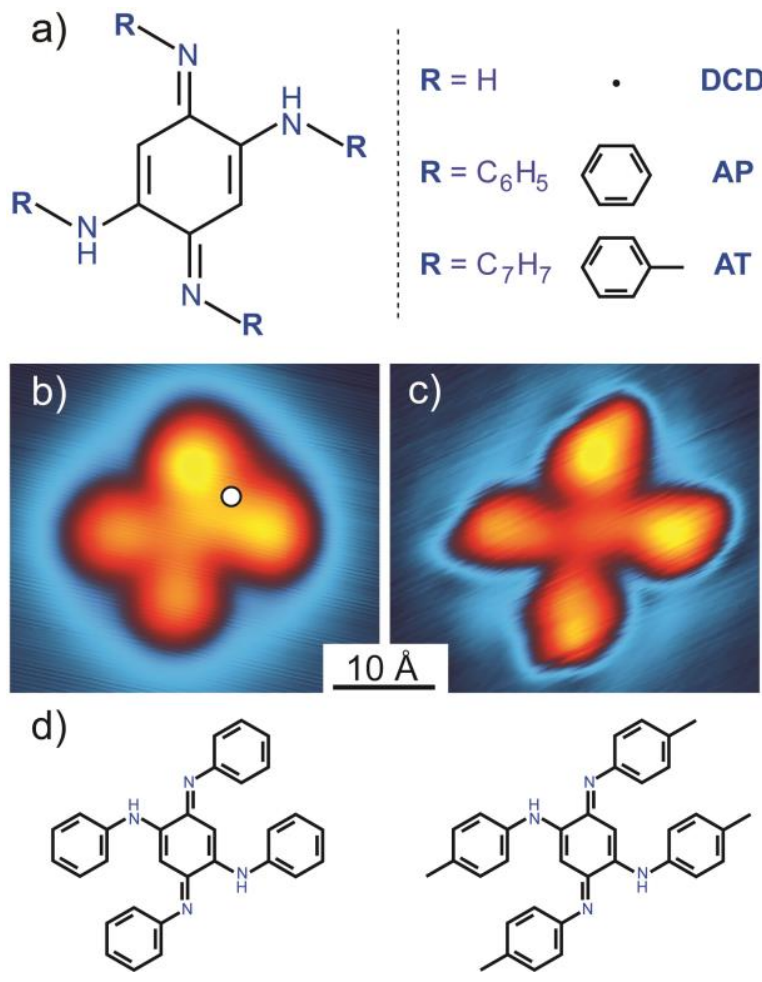

Figure 1: Chemical structures of DCD, AP, and AT; STM topographies of AP and AT. (a) Central functional unit common to all three molecules. $\mathrm{R}$ substituted with the relevant side groups to form $D C D, A P$, or AT. (b) STM image of AP (I = $1.00 \mathrm{nA}, \mathrm{V}=-0.19 \mathrm{~V})$ with dot indicating the site of manipulation. (c) STM image of AT ( $I=0.03 \mathrm{nA}$, $\mathrm{V}=-0.06 \mathrm{~V}$ ). (d) Chemical structures of the respective compounds in the native para form.

In a first step, the gas phase geometries and energies for $D C D$ and $A P$ were investigated using DFT calculations both in the absence and the presence of $\mathrm{Cu}$ species (see Supporting Information S1). The para, ortho, and meta configurations were optimized for each molecule and found to adopt highly nonplanar conformations. We find that the para configuration is favored in the absence of $\mathrm{Cu}$ species, but the ortho configuration is favored in their presence, in agreement with previous experimental studies. ${ }^{17,22}$ Further to this, the transition states for para to meta configurational changes were also computed (the reasons for this will become clear later) and located at values of 0.959 and $0.795 \mathrm{eV}$ for $D C D$ and $A P$, respectively.

With this proton transfer property in mind, and the molecules' affinity for copper, $\mathrm{Cu}(110)$ was chosen as a substrate to investigate these molecules as a possible system for supported molecular switching. Besides copper being ubiquitous in the fabrication of electronic components, both the chosen surface and the molecules have 2-fold symmetry (assuming planar molecular adsorption configurations), and so the number of possible adsorption arrangements is limited, reducing the complexity of analysis. The molecules were deposited onto the $\mathrm{Cu}(110)$ substrate held at $100 \mathrm{~K}$ for both AP and AT in order to achieve a clean surface decorated with isolated molecules. This constitutes an important criterion if we wish to address them on an individual basis and ensure that species adsorbed in the local environment do not affect any potential switching behavior. After preparation, samples were cooled further to $5 \mathrm{~K}$ before imaging using STM in constant current mode. To date, very few experimental studies and no STM studies have been conducted on these molecules despite AP first being synthesized in $1875 .^{23}$

Our STM data show that the molecules have indeed adsorbed isolated from one another on the terraces of the $\mathrm{Cu}(110)$ substrate. The AP and AT molecules shown in Figure 1b,c, respectively, exhibit topographies that are clearly recognizable with respect to their chemical structures. The data show molecules with four lobes each corresponding to the expected four peripheral phenyl or tolyl groups. When comparing the shape of the lobes in these two molecules, we recognize that the lobes of AT are slightly more pointed at the edges compared to AP, indicating the presence of the extra methyl groups. From this, we deduce that both AP and AT have adsorbed in flat-lying geometries, accommodated by rotations of the side groups upon interaction with the substrate.

In addition, a number of different adsorption configurations of AP and AT are seen. These include higher energy buckled and metastable species, as well as the lowest energy adsorption geometries shown in Figure 1b,c. In this letter, only the energetically lowest and most stable species are considered. These can be identified after simple annealing experiments, in which the temperature of the sample is raised followed by recooling to $5 \mathrm{~K}$, whereby only one type of AP species remains. A full description of this and examination of the higher 
energy species observed on the surface can be found in Supporting Information S2.

Another feature common to both lowest energy AP and $A T$ is that two adjacent lobes show similar apparent STM heights. Specifically, we have two lobes with bright contrast opposite two lobes with dim contrast. In the first instance, this contradicts the expected topography of diagonally opposite lobes sharing similar electronic signatures due to the molecules occupying the para state natively. We therefore infer that a conformational change has occurred upon adsorption. For both molecules, two possibilities exist: para configurational change to either ortho or meta. As discussed earlier, a para-AP to ortho-AP change is favored in the presence of copper and a similar change for AT is hence also likely. Despite no Lewis structure for the gas-phase meta conformers existing, the metal surface could stabilize such structures. ${ }^{24}$ The chemical insensitivity of STM does not allow us to identify straightforwardly, which conformer is stabilized by the substrate. However, we will later unambiguously show by DFT calculations that the presence of meta conformers is energetically preferred.
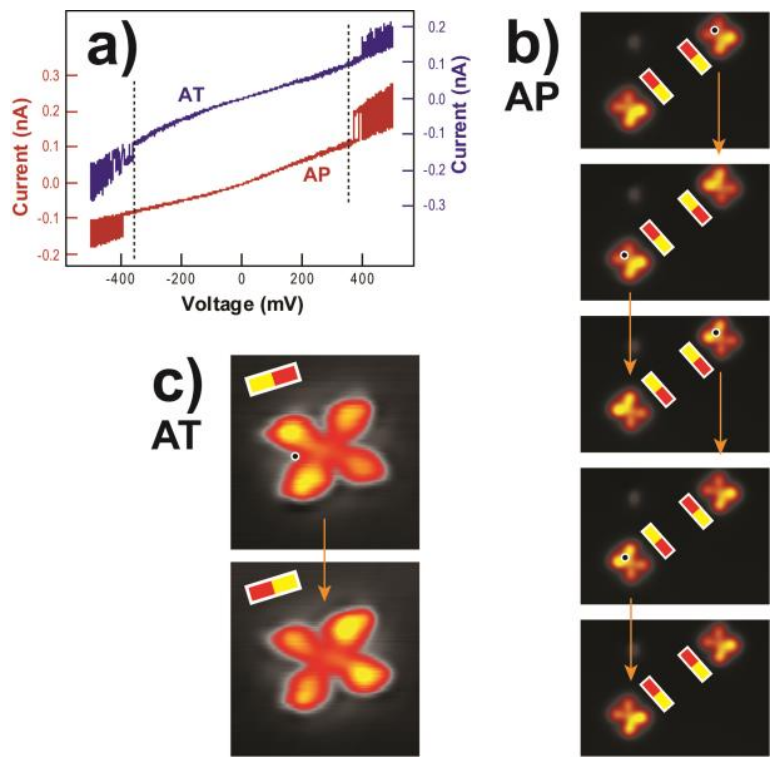

Figure 2: Demonstration of switching in AP and AT. (a) IV characteristics for AP (red trace) and AT (blue trace). (b) STM images of neighboring AP molecules $(I=1.00 \mathrm{nA}, \mathrm{V}=$ $-0.19 \mathrm{~V})$. The marked point in each image shows the site of a $-0.35 \mathrm{~V}$ pulse to switch the molecule, and the next image in the sequence displays the outcome of the manipulation. (c) Demonstration of switch in AT ( $=0.03 \mathrm{nA}, \mathrm{V}=-0.06 \mathrm{~V})$.

When comparing AP and AT adsorbed on the surface, we see that there are four possibilities for the positions of the bright lobes, each corresponding to a $90^{\circ}$ rotation with respect to the underlying substrate. Statistical analysis of hundreds of observed molecules shows that these four adsorption states are equally populated. The possibility of switching between these states in individual molecules using tunneling electrons was therefore investigated. For both AP and AT, the STM tip was brought to an off-center symmetric position between two of the lobes sharing an identical contrast, as indicated in Figure 1c. The feedback electronics were then deactivated, and the bias swept from -0.5 to $+0.5 \mathrm{~V}$ while measuring the tunnelling current between tip and sample. From Figure $2 a$, we see a featureless curve for both molecules between -0.35 and $+0.35 \mathrm{~V}$ indicating that no switching is taking place. Outside this interval, a sharp, rapid switching of the current indicates that the molecules are switching. A further manipulation in the same position, but with a constant bias voltage outside the $\pm 0.35 \mathrm{~V}$ range, reveals that the current switches between two distinct values. This property fulfills the criteria for a molecular switch because the binary behavior corresponds to the molecules alternatively accessing two different conductance states.

The topographic change in these molecules accompanying this switching process is demonstrated by means of sequential STM imaging. Figure $2 b$ shows two neighboring AP molecules occupying the same state. The tip is then brought to the indicated position, the feedback deactivated, and the bias raised to a value above the switching threshold. After a sharp change in the tunnel current is observed, the manipulation is stopped, and the same area is imaged to reveal that the targeted molecule has adopted another state in which the bright lobes occupy the opposite positions compared to the original state. The neighboring molecule remains unchanged indicating that the stimulus is localized to the position directly below the STM tip. The series of consecutively acquired images in Figure $2 \mathrm{~b}$ shows both molecules being switched back-andforth by means of controlled voltage pulses. Importantly, when addressing a single molecule, the switching process involves only two (out of the possible four) states that possess opposite bright and dim lobes. A switching mechanism involving a $90^{\circ}$ rotation of the bright and dim lobes is never observed. We therefore have two separate but equivalent meta species distinguished by a $90^{\circ}$ rotation to each other. Each of these meta species has two states corresponding to changes in contrast of opposite pairs of lobes when measured using STM. The observed switching is hence equivalent to a $180^{\circ}$ rotation; however, physical rotation of the molecule can be ruled out due to the identical switching behavior being observed within molecular islands of AP (see Supporting Information S3). Steric 
hindrance in such an environment precludes any rotational motion and therefore points to a mechanism involving an internal change in the molecule.

The same switching behavior is present in the further functionalized AT molecule, as shown in Figure 2c. An identical manipulation was repeated on this molecule to achieve the same qualitative result of moving the STM contrast from one pair of lobes to the opposite two. We also see in these images that the shapes of the peripheral groups do not change after the switch. This rules out a possible conformational change due to phenyl or tolyl rotations within the molecules. At this stage we have shown that AP and AT are controllably switchable and exhibit the same switching behavior. In addition, we note from Figure $2 a$ that the threshold voltages for switching AT and AP are comparable (within experimental errors). It can therefore be said that chemical functionalization of AP with additional methyl groups (to form AT) does not affect the switching property. The excitation process and mechanism, however, need to be addressed. A full study of the switching mechanism was conducted on $A P$, and the remainder of this letter will focus on that.

To identify the excitation process governing the observed switching of AP, many manipulation events were recorded as the tunnel current, I, and bias voltage, $\mathrm{V}$, were varied. These measurements were carried out on isolated molecules to eliminate any influence of species in the local environment. ${ }^{25}$ The tip was positioned as indicated in Figure $2 b$ for all measurements to ensure that the measured tunnel current had two distinguishable values (if a more central position is chosen, the molecule still switches, but the current has a single value due to small change in electronic height). To measure the switch rate at any given I and V, the time intervals in which the molecule occupied the high current state were binned and plotted in a histogram. The distribution of residence times takes the form of an exponential decay as shown in Figure 3a, indicating that the switch is a spontaneous chemical process and that the molecule has no memory of what state it occupies. The inverse time constant from the exponential fit gives the switch rate at that particular $\mathrm{I}$ and $\mathrm{V}$. The relationship between tunnel current and switch rate was recorded by repeating the above process at many different values of $\mathrm{I}$, while $\mathrm{V}$ was held constant (Figure 3b). For voltages exceeding $\pm 0.35 \mathrm{~V}$ the relationship is linear, indicating that the switching is triggered by a single electron inelastically scattered by the molecule. ${ }^{26}$
The switch rate as a function of tunnel current remains linear until the bias voltage is dropped within the $\pm 0.35 \mathrm{~V}$ range, after which the switch rate was found to be proportional to $I^{N}$, where $N$ is the number of inelastic electrons activating the process. As seen in Figure $3 c$, below this threshold voltage, $N$ increases as the electron energy decreases, indicating a vibrational ladder climbing process activated by multiple electrons. The dependence of $N$ with $V$ indicates that the lowest energy required by a single electron and hence the activation energy of the switch is $0.35 \mathrm{eV}$.
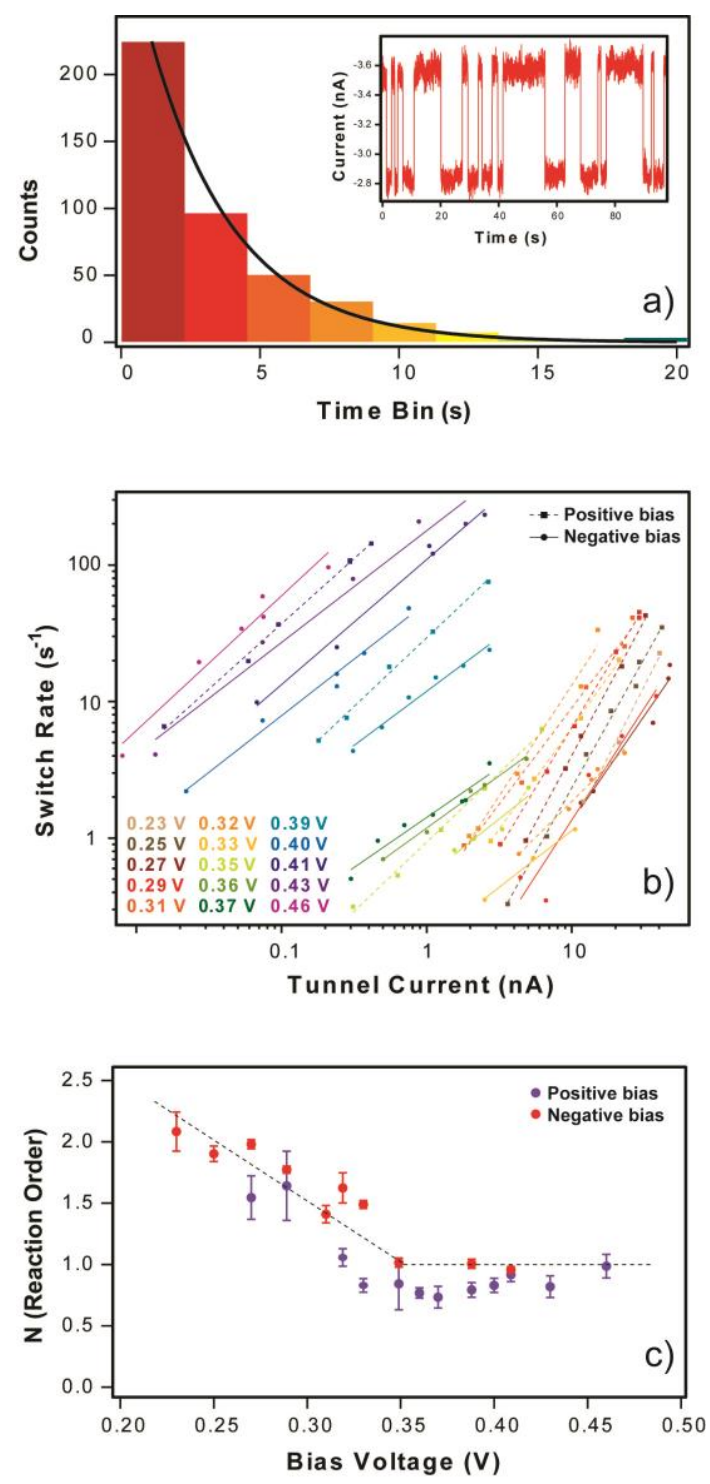

Figure 3: Switching statistics of AP. (a) Typical distribution of the high-current residence times from current versus time measurements (inset). Bias voltage is held at $-0.25 \mathrm{~V}$, while the high current value is measured as $3.6 \mathrm{nA}$. The rate of switching at this $\mathrm{I}, \mathrm{V}$ is extracted from the exponential fit (black curve) to the data. (b) Switch rate as a function of tunnel current for a range of biases. Fits to the data are power laws of the form rate $\propto$ IN. (c) Variation of $\mathrm{N}$, the reaction order, with $\mathrm{V}$. 
After establishing that the switching process is activated by inelastic tunneling electrons and in order to fully describe this molecular system, we now turn to determining the switching mechanism (a proof for $\mathrm{H}$-tautomerism) by comparing our experimental observations with theoretical calculations. Periodic DFT geometry optimizations were carried out for para-AP, ortho-AP, and meta-AP adsorbed on a $\mathrm{Cu}(110)$ slab. As shown in Figure 4 (and detailed in Supporting Information S4), all optimized configurations have a number of common points: (1) the 4-fold coordination site of the $\mathrm{Cu}(110)$ surface acts as adsorption center; and (2) the two main molecular axes (through diagonally opposite lobes) of AP align with the [110] and [001] high symmetry directions of the Cu substrate. In addition, each para-, ortho-, and meta-AP conformer can adsorb in four possible configurations (illustrated for meta and para in Supporting Information S4). Symmetry arguments (and our DFT calculations) show that these four configurations for ortho- and meta-AP are energetically equivalent. In para-AP, however, identical (amino or imino) groups can either lie parallel or perpendicular to one of the high-symmetry directions of the surface, giving rise to two nondegenerate states (each consisting of two configurations). Contrary to the gas-phase calculations, meta-AP adopts the lowest energy structure, followed by para-AP (less stable by 0.15 and $0.25 \mathrm{eV} / \mathrm{molecule}$ ) and then ortho-AP (less stable by $1.02 \mathrm{eV} /$ molecule; we discard ortho-AP henceforth on the basis of these energetic arguments). Using the Tersoff-Hamann approach, ${ }^{27,28}$ STM images for both (lowest energy) para- and meta-AP/Cu(110) were simulated using the optimized structures. States between the Fermi energy $\left(E_{\mathrm{f}}\right)$ and $\left(E_{\mathrm{f}}-0.2 \mathrm{eV}\right)$ were integrated to simulate our typical tunnelling conditions, and the resultant images are displayed in Figure $4 a, b$.

Only meta-AP possesses two adjacent lobes that share a similar bright contrast, matching our experimental observations. In addition, the experimental determination of the precise adsorption geometry (procedure described in Supporting Information S5) matches the DFT predictions, in which the central ring of the molecule lies in the 4-fold coordination site of $\mathrm{Cu}(110)$ with the molecular axes aligning with the high symmetry [110] and [001] directions. The unpaired electrons, which would be present in the isolated meta configuration, are likely stabilized by donation or acceptance from the surface. ${ }^{24}$ On the basis of the excellent agreement between theory and experiments, we hence unambiguously demonstrate that AP adsorbs in the meta form. From this, we can assign the location of the amino $\mathrm{H}$ atoms to the position of the bright lobes, and conversely, the imino groups are associated with the dim lobes in the STM contrast. This proves that the observed switching mechanism is a consequence of tautomerization of the $\mathrm{H}$ atoms from the amino groups to the adjacent imino groups. This tautomerization is accompanied by electronic reordering of the molecule, which is manifested in the STM images as a shift in brightness (apparent height) from one side of the molecule to the other.
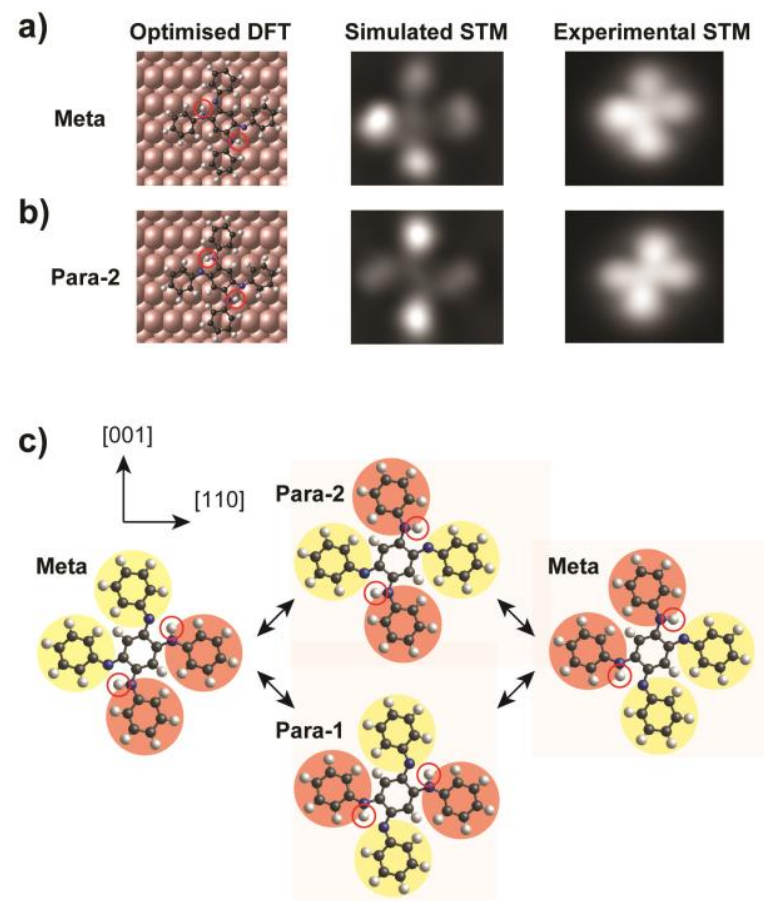

d)

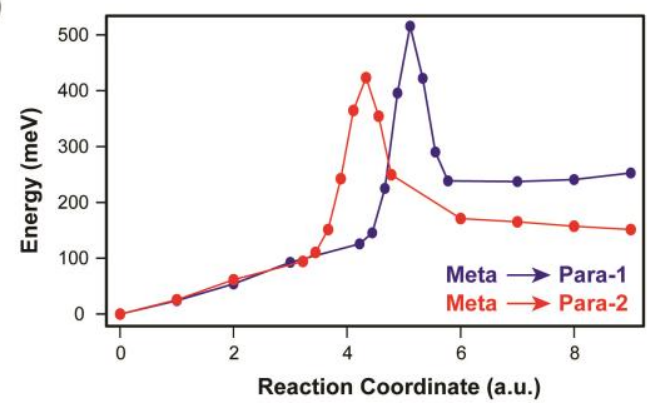

Figure 4: Switching mechanism in AP. (a) DFT optimized structure of meta-AP alongside simulated and experimental STM images $(I=2.40 \mathrm{nA}, \mathrm{V}=-0.18 \mathrm{~V})$. (b) DFT optimized structure of para-AP intermediate with simulated and experimental STM images $(I=2.20 \mathrm{nA}, \mathrm{V}=$ $-0.18 \mathrm{~V})$. (c) Reaction pathways for meta-to-meta transition. The para intermediates are nondegenerate due to the symmetry of the surface. (d) Potential energy diagram calculated using nudged elastic band method shows that para-2 is the lowest energy, favoured intermediate.

To address the reaction pathway through which the molecule undergoes $\mathrm{H}$-tautomerization, we concentrate on a single meta-to-meta transition (the 
argument is valid for the other equivalent adsorption configuration rotated by $90^{\circ}$ ). Two possible reaction mechanisms can be conceived: a concerted doubleproton transfer or a sequential single-proton transfer. DFT-based nudged elastic band (NEB) calculations (as detailed in Supporting Information S4) for the concerted double-proton mechanism indicate that, rather than a single activation barrier, there exist two sequential activation barriers, pointing to the presence of a two-step process. We hence focus on the sequential process, whereby the first proton can be transferred from one of two possible amino groups. This gives rise to two inequivalent para-AP intermediate states distinguished by the alignment of their amino groups either parallel or perpendicular to the [110] Cu rows, as shown in Figure 4c. The corresponding NEB calculations (Figure $4 d$ ) reveal that the activation barriers in achieving these two states are different. The lowest energy and hence favored intermediate has an activation energy of $0.42 \mathrm{eV}$ (in qualitative agreement with the $0.35 \mathrm{eV}$ barrier experimentally determined, noting that the generalized gradient approximation, or GGA, tends to overestimate barriers), whereas the higher energy pathway requires $0.52 \mathrm{eV}$. Once the reaction intermediate is formed, the molecule then quickly relaxes into one of the two equally possible meta states corresponding to transfer of the second proton to the adjacent imino group (molecule has switched), or return of the first proton to its original position (molecule remains unchanged). In rare circumstances (below 1\%), the lowest energy reaction intermediate is stabilized on the surface during a typical switching procedure and can be seen in Figure $4 \mathrm{~b}$ alongside its DFT simulated image.

We finally revert to our initial gas phase DFT calculations. Therein, the transition state for para- to meta-AP transformation was computed at $0.795 \mathrm{eV}$. This represents a sizable difference when compared to the activation energy experimentally measured $(0.35 \mathrm{eV})$ or theoretically computed for the $\mathrm{AP} / \mathrm{Cu}(110)$ system $(0.42 \mathrm{eV})$. We hypothesize that, upon deposition, the resulting molecular distortion to an adsorbed planar and meta conformation (by rotation of the phenyl groups) leads to a significant lowering of the activation energy as a consequence of a strong interaction with the Cu surface. We note that meta-AP adopting the lowest energy adsorption structure is nevertheless surprising because, in isolation, no Lewis structure exists for this isomer. However, through interaction with the substrate, the calculations clearly show that this structure is stabilized and energetically preferred over para-AP and ortho-AP. This change in configuration, as we understand, is a key element that enables/retains the $\mathrm{H}$-tautomeric switching property in our $\mathrm{Cu}(110)$ bound quinone derivatives. Indeed, in addition to the fact that the three-dimensional active moiety (the two imino and two amino groups) is rendered two-dimensional upon interaction with the substrate, the distance over which the $\mathrm{H}$ atoms are transferred from amino to imino groups is reduced in the stabilized meta state when compared to the previously expected ortho configuration (favored in the gas phase when in the presence of a Cu species).

In summary, a new class of molecular switches, which display bistability in conductance due to $\mathrm{H}$ tautomerism, has been realized. AP and AT adsorbed on $\mathrm{Cu}(110)$ produce stable molecular switches, which can be activated on demand using voltages above a specific threshold $( \pm 0.35 \mathrm{eV})$. The switching behavior in both AP and AT shows the same characteristics, and although the same analytical rigor was not applied to AT, the adsorption registry, excitation process, and reaction mechanism are likely the same as that proved for AP. The trigger for a switching event is a single inelastically scattered electron, and by probing a range of electron energies, it was found that the minimum energy required for $A P$ and $A T$ is $0.35 \mathrm{eV}$, and our computational results support this $(0.42 \mathrm{eV}$ for $\mathrm{AP})$. Further computational modeling confirms that the observed behavior is due to a two-step $\mathrm{H}$ atom transfer from the amine to the imine functional constituent groups. H-tautomeric molecular switches to date have only involved the movement of protons locked into a protective porphyrin macrocycle. The present data, however, reveal that this mechanism can likely be generalized to many other molecules and does not need to be restricted to this moiety. The constraints for devising surface-bound $\mathrm{H}$ tautomeric switches (undeformed, protective, twodimensional active unit) can now be relaxed. We can therefore contemplate more versatile molecular candidates, as we have shown here with quinone derivatives.

Most importantly, the presence of the equivalent switching mechanism in AT means that functionalization of the central switching unit does not compromise this property nor does it induce measurable changes in the activation energy. This shows that functionalization of the original AP molecule (albeit with simple methyl groups) paves the way for novel opportunities toward further modification of the chemical structure to achieve a molecular switch with specific, tailored properties. Molecules with thiol functional groups that chemisorb strongly to metal electrodes, for instance, could facilitate incorporation into nanoelectrical circuits. Alternatively, coordination chemistry could 
enable insertion into molecular architectures. A functional molecular switch may be considered to be a molecule that has two stable states and does not radically change its position or shape when integrated into a circuit. The examples developed in this letter show both of these attributes and thus may be considered useful as a starting point for the development of molecular based nanoelectronic devices.

\section{ASSOCIATED CONTENT}

Supporting Information: Details of UHV STM system. DFT calculations of gas phase and surface bound molecules. Observation of switching in a molecular island. Explanation of adsorption configurations of AP and AT. Adsorption registry determination of AP. Details of synthesis of AP. This material is appended at the end of this document.

\section{ACKNOWLEDGEMENTS}

R.S. acknowledges the financial support from the Scottish Funding Council through EaStCHEM and SRDG grant HR07003. We acknowledge Profs WolfDieter Schneider, Flemming Besenbacher, and Neville Richardson for fruitful discussions and critical reading of the manuscript. Calculations were carried out on the EaStCHEM Research Computing Facility and the UK national supercomputing service HECTOR.

\section{REFERENCES}

(1) Liang, W.; Shores, M. P.; Bockrath, M.; Long, J. R.; Park, H. Nature 2002, 417, 725-729.

(2) Aviram, A.; Ratner, M. A. Chem. Phys. Lett. 1974, 29, 277-283.

(3) Martin, A. S.; Sambles, J. R.; Ashwell, G. J. Phys. Rev. Lett. 1993, 70, 218-221.

(4) Moresco, F.; Meyer, G.; Rieder, K.-H.; Tang, H.; Gourdon, A.; Joachim, C. Phys. Rev. Lett. 2001, 86, 672-675.

(5) Emberly, E.; Kirczenow, G. Phys. Rev. Lett. 2003, 91, 1-4.

(6) Leoni, T.; Guillermet, O.; Walch, H.; Langlais, V.; Scheuermann, A.; Bonvoisin, J.; Gauthier, S. Phys. Rev. Lett. 2011, 106, 4-7.

(7) Comstock, M.; Levy, N.; Kirakosian, A.; Cho, J.; Lauterwasser, F.; Harvey, J.; Strubbe, D.; Fréchet, J.; Trauner, D.; Louie, S.; Crommie, M. Phys. Rev. Lett. 2007, 99, 1-4.

(8) Qiu, X. H.; Nazin, G. V.; Ho, W. Phys. Rev. Lett. 2004, 93, 1-4.

(9) Ohmann, R.; Vitali, L.; Kern, K. Nano Lett. 2010, 10, 2995-3000.

(10) Swart, I.; Sonnleitner, T.; Repp, J. Nano Lett. 2011, 11, 1580- 1584.

(11) Choi, B.-Y.; Kahng, S.-J.; Kim, S.; Kim, H.; Kim, H.; Song, Y.; Ihm, J.; Kuk, Y. Phys. Rev. Lett. 2006, 96, 1-4.

(12) Alemani, M.; Peters, M. V.; Hecht, S.; Rieder, K.-H.; Moresco, F.; Grill, L. J. Am. Chem. Soc. 2006, 128, 14446-14447.
(13) Morgenstern, K. Acc. Chem. Res. 2009, 42, 213-223.

(14) Liljeroth, P.; Repp, J.; Meyer, G. Science 2007, 317, 1203-1206.

(15) Auwärter, W.; Seufert, K.; Bischoff, F.; Ecija, D.; Vijayaraghavan, S.; Joshi, S.; Klappenberger, F.; Samudrala, N.; Barth, J. V. Nat. Nanotechnol. 2012, 7, 41-46.

(16) Frantz, S.; Rall, J.; Hartenbach, I.; Schleid, T.; Zális, S.; Kaim, W. Chem. Eur. J. 2004, 10, 149-154.

(17) Rall, J.; Stange, A. F.; Hübler, K.; Kaim, W. Angew. Chem., Int. Ed. 1998, 37, 2681-2682.

(18) Huang, Y.-B.; Tang, G.-R.; Jin, G.-Y.; Jin, G.-X. Organometallics 2008, 27, 259-269.

(19) Ujike, K.; Kudoh, S.; Nakata, M. Chem. Phys. Lett. 2005, 409, 52-56.

(20) Rumpel, H.; Zachmand, G. J. Phys. Chem. 1989, 93, 1812-1818.

(21) Rumpel, H.; Limbach, H. H. J. Am. Chem. Soc. 1989,

111, 5429-5441.

(22) Braunstein, P.; Demessence, A.; Siri, O.; Taquet, J.-P. C. R. Chim. 2004, 7, 909-913.

(23) Kimich, C. Ber. Dtsch. Chem. Ges. 1875, 8, 1026-1032.

(24) Bieri, M.; Nguyen, M.-T.; Gröning, O.; Cai, J.; Treier, M.; Aitt-Mansour, K.; Ruffieux, P.; Pignedoli, C. A.; Passerone, D.; Kastler, M.; Müllen, K.; Fasel, R. J. Am. Chem. Soc. 2010, 132, 16669-16676.

(25) Kumagai, T.; Hanke, F.; Gawinkowski, S.; Sharp, J.; Kotsis, K.; Waluk, J.; Persson, M.; Grill, L. Nat. Chem. 2014 6, 41-46.

(26) Stipe, B. C.; Rezaei, M. A.; Ho, W. Science 1998, 279, 1907-1909.

(27) Tersoff, J.; Hamann, D. R. Phys. Rev. Lett. 1983, 50, 1998-2001.

(28) Tersoff, J.; Hamann, D. R. Phys. Rev. B 1985, 31, 805-813. 


\title{
Supplementary Information
}

\section{A new class of metal bound molecular switches involving $\mathrm{H}$-tautomerism}

\author{
Grant J. Simpson ${ }^{1}$, Simon W. L. Hogan ${ }^{1}$, Marco Caffio ${ }^{1}$, Christopher J. Adams ${ }^{2}$, Herbert \\ Früchtl $^{1}$, Tanja van Mourik ${ }^{1}$ and Renald Schaub ${ }^{1 *}$ \\ 1 EaStCHEM and School of Chemistry, University of St Andrews, St Andrews, KY16 9ST, United \\ Kingdom \\ 2 School of Chemistry, University of Bristol, Bristol, BS8 1TS, United Kingdom \\ * email: renald.schaub@st-andrews.ac.uk
}

\section{Methods}

All experiments were performed using a commercially available STM (Createc GmbH) operated at $5 \mathrm{~K}$ housed in a UHV chamber. During measurements the pressure in the UHV chamber was below $4 \times 10^{-11} \mathrm{mbar}$. The $\mathrm{Cu}(110)$ single crystal (MaTeck $\mathrm{GmbH}$ ) was cleaned by repeated cycles of sputtering with $\mathrm{Ar}^{+}\left(1.5 \mathrm{keV}, 1 \times 10^{-6} \mathrm{mbar}, 10 \mathrm{~min}\right)$ followed by annealing to $773 \mathrm{~K}$ ( $15 \mathrm{~min}$ ). Before deposition, the sample was cooled to approximately $78 \mathrm{~K}$ using liquid nitrogen. Molecules were deposited on the cleaned surface by sublimation from a glass tube heated by a filament held at $396 \mathrm{~K}$ and $406 \mathrm{~K}$ for AP and AT, respectively. AP was synthesized according to the methods in SuppIMat S6, and AT was purchased from SigmaAldrich. The STM images presented were collected constant current mode and the current/time data was collected using the open feedback mode after the tunnel parameters were set. A tungsten tip which had been electrochemically etched using a solution of potassium hydroxide was used. Igor Pro was used for all data and image analysis.

\section{S1. Gas-phase calculations on AP and DCD}

The geometries of AP and the equivalent molecule with phenyl rings replaced by hydrogen atoms (3,6-diimino-1,4-cyclohexadiene-1,4-diamine; hereafter abbreviated to DCD) were optimized in the gas phase using density functional theory (DFT) with the M06-2X functional and the $6-31+G(d)$ basis set. The calculations were performed with the Gaussian 09 program $^{2}$. The structures were also optimized in the presence of a neutral as well as positively charged $\mathrm{Cu}$ atom.

\section{Isolated $A P$ and $D C D$}


Gas-phase AP is highly non-planar and can adopt different conformations. Figure S1 shows the most stable conformations found for the para, meta and ortho forms of the two molecules. Para-AP is energetically favored, followed by meta-AP (with an energy of 0.548 $\mathrm{eV}$ relative to para-AP) and ortho-AP (relative energy: $0.798 \mathrm{eV}$ ). The relative stability order of DCD differs from that of AP: the para form is still the energetically most favorable, but ortho-DCD is more stable than meta-DCD (relative energies: ortho: $0.420 \mathrm{eV}$; meta: 0.635 $\mathrm{eV})$. The ortho tautomers are disfavored because of steric hindrance of the two amino hydrogens. In DCD, this is partially offset by the formation of an intramolecular $\mathrm{N}-\mathrm{H} \bullet \bullet \mathrm{N}$ contact, with the result that ortho-DCD is energetically favored compared to meta-DCD. The transition states for para $\rightarrow$ meta tautomerisation were also located. For $\mathrm{AP}$, this transition state lies $0.795 \mathrm{eV}$ above the para tautomer, whereas for DCD it lies $0.959 \mathrm{eV}$ above the para structure. Figure S2 shows the relative energies of the stationary points along the para $\rightarrow$ meta tautomerisation pathway of the two molecules.

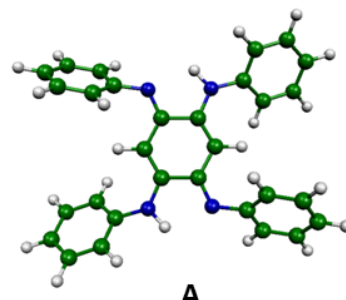

A

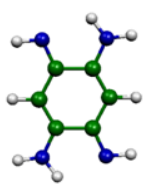

D

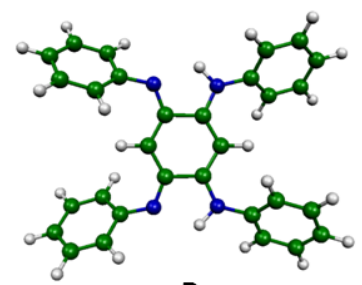

B

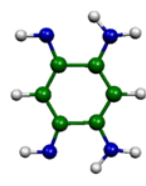

E

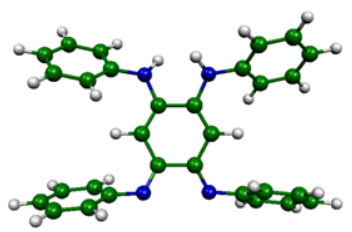

C

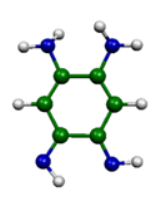

$\mathbf{F}$

Figure S1 The para, meta and ortho forms of $A P(A, B$ and $C$, respectively) and of $D C D$ (D, E and F, respectively). All three AP tautomers have $C_{2}$ symmetry. In para-AP the $C_{2}$ axis is perpendicular to the central ring and goes through its midpoint. In the ortho tautomer the $C_{2}$ axis is in the plane of the central ring and crosses the middle of the (N-)C-C(-N) bonds. In meta-AP the $\mathrm{C}_{2}$ axis goes through the ( $\left.\mathrm{H}-\right) \mathrm{C}$ atoms of the central ring. Ortho-DCD does not have any symmetry elements. Para-DCD has $\mathrm{C}_{2 \mathrm{~h}}$ symmetry, whereas meta-DCD has $\mathrm{C}_{2 \mathrm{v}}$ symmetry. 


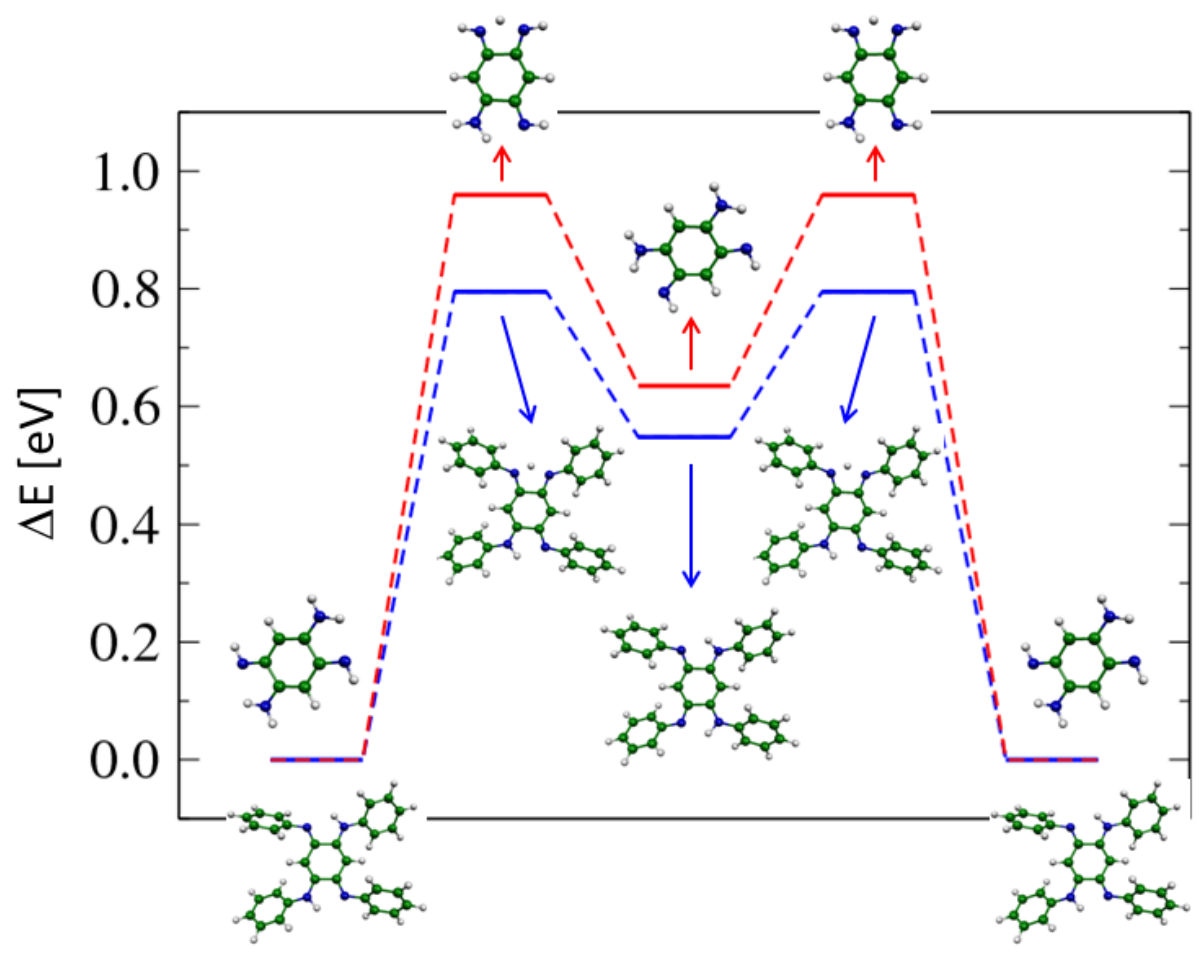

Figure S2 Relative energies (in eV) of the stationary points along the para $\rightarrow$ meta tautomerisation pathway of AP (blue) and DCD (red), calculated with M06-2X/6-31+G(d).

The barrier for para $\rightarrow$ meta tautomerisation was previously computed at the HF/3-21G and MP2/3-21G//HF/6-31G levels for DCD by Holloway ${ }^{3}$. The MP2/3-21G value of $0.57 \mathrm{eV}$ is much smaller than the M06-2X/6-31+G(d) value calculated here $(0.96 \mathrm{eV})$. Considering the very limited basis set used in the earlier calculations, the M06-2X value is expected to be more realistic.

\section{2. $A P$ and $D C D$ with $C u$ and $C U^{+}$}

Using the B3LYP density functional, Kaim et al. ${ }^{4}$ showed that in the presence of $\mathrm{a} \mathrm{Li}^{+}$or $\mathrm{Cu}^{+}$ ion the ortho form of DCD is preferred over the para form by 0.38 and $0.37 \mathrm{eV}$, respectively. With M06-2X, the relative energy of the para tautomer with $\mathrm{Cu}^{+}$is $0.33 \mathrm{eV}$ (Figure S3). As far as we are aware, AP with $\mathrm{Cu}^{+}$has not yet been studied computationally. We calculate a relative energy of $0.23 \mathrm{eV}$ for para-AP. The meta tautomer of AP and DCD is much more disfavored in the presence of $\mathrm{Cu}^{+}$, with relative energies of $1.53 \mathrm{eV}(\mathrm{DCD})$ and $0.97 \mathrm{eV}(\mathrm{AP})$. We have also optimized the structures in the presence of a neutral $\mathrm{Cu}$ atom. The optimized structures are displayed in Figure S4. Qualitatively, the results are similar to those for $\mathrm{Cu}^{+}$: The ortho tautomer is most stable, whereas the meta tautomer is most disfavored energetically. In the ortho structures, the $\mathrm{Cu} / \mathrm{Cu}^{+}$is equidistant to the coordinating $\mathrm{N}$ atoms and located in the plane of the central ring, preserving the $\mathrm{C}_{2}$ symmetry of the AP structure. In ortho-DCD, the presence of the $\mathrm{Cu}$ or $\mathrm{Cu}^{+}$species induces $\mathrm{C}_{2}$ symmetry. The $\mathrm{Cu} / \mathrm{Cu}^{+} \bullet \bullet N$ distances range from 2.0-2.1 $\AA$. In the para structures, the presence of $\mathrm{Cu}_{\text {or }} \mathrm{Cu}^{+}$removes 
any symmetry elements, though para-DCD-Cu is nearly $\mathrm{C}_{\mathrm{s}}$-symmetric (due to the nearplanar amino group). The $\mathrm{Cu} / \mathrm{Cu}^{+}$is slightly closer to the imino than amino nitrogen and slightly out of the plane of the central ring, with distances to the imino $\mathrm{N}$ of about $2.0 \AA$ and to the amino $\mathrm{N}$ of about $2.1 \AA$. Also in the meta structures the $\mathrm{Cu} / \mathrm{Cu}^{+}$removes all symmetry elements present in the structures without metal atoms. The $\mathrm{Cu} / \mathrm{Cu}^{+}$is also closer to the imino than amino nitrogen and located out of the plane of the central ring, but the differences are slightly larger than for the para tautomers $\left(\mathrm{Cu} / \mathrm{Cu}^{+} \bullet \bullet N\right.$ (imino): 1.9-2.0 $\AA$; $\mathrm{Cu} / \mathrm{Cu}^{+} \bullet \bullet N$ (amino): 2.2-2.3 $\AA$ ). The biggest differences occur for meta-DCD-Cu ${ }^{+}$, where the two $\mathrm{Cu}^{+} \bullet \bullet N$ distances are 1.93 and $3.34 \AA$. In this structure the $\mathrm{Cu}^{+}$is far out of the plane of the ring (by about $45^{\circ}$ ). In the other meta structures, the $\mathrm{Cu} / \mathrm{Cu}^{+}$is out of the plane of the central plane by about $10-20^{\circ}$. In summary, these results show that the presence of a $\mathrm{Cu}$ atom or $\mathrm{Cu}^{+}$ion changes the relative stability of the AP and DCD tautomers considerably.

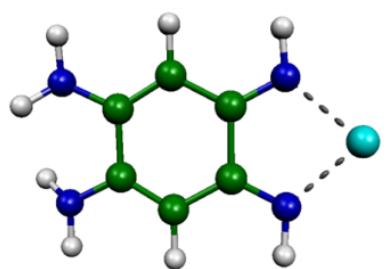

$0-\mathrm{DCD}(0.000 \mathrm{eV})$

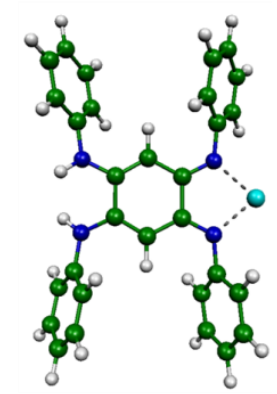

o-AP $(0.000 \mathrm{eV})$

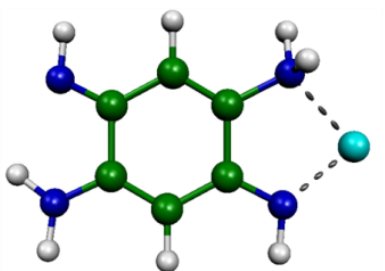

$p-\operatorname{DCD}(0.326 \mathrm{eV})$

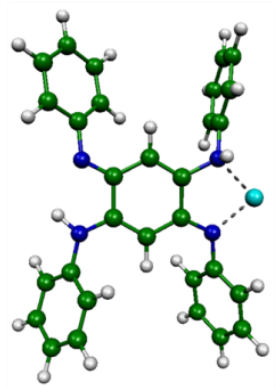

$p-\operatorname{AP}(0.234 \mathrm{eV})$
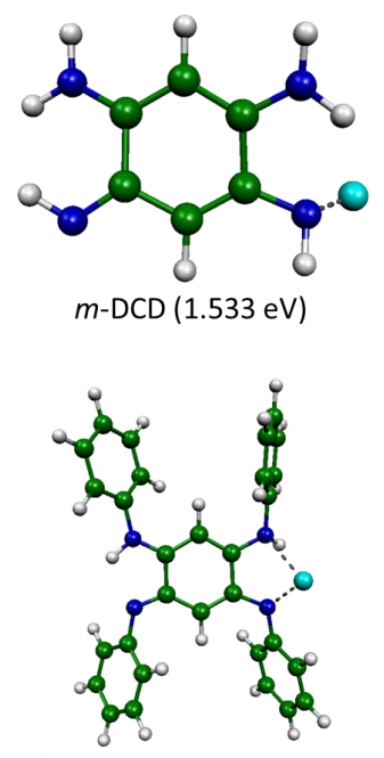

$m-\mathrm{AP}(0.969 \mathrm{eV})$

Figure S3 M06-2X/6-31+G(d) optimized structures of DCD and AP in the presence of $\mathrm{Cu}^{+}$. Energies relative to the ortho tautomer are given in parentheses. 




$0-\mathrm{DCD}(0.000 \mathrm{eV})$

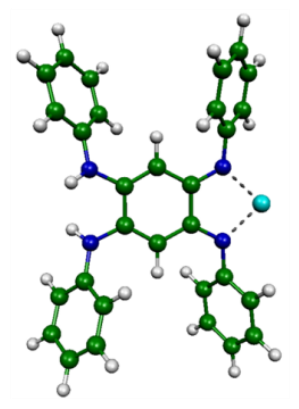

$0-\mathrm{AP}(0.000 \mathrm{eV})$

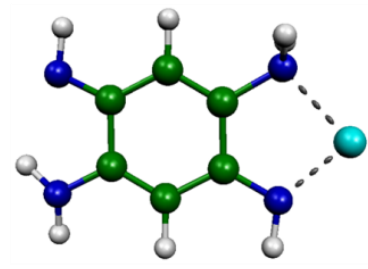

$p-\operatorname{DCD}(0.549 \mathrm{eV})$

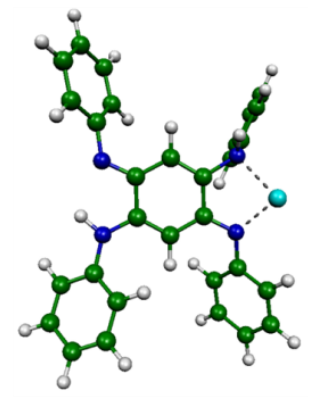

$p-\operatorname{AP}(0.362 \mathrm{eV})$

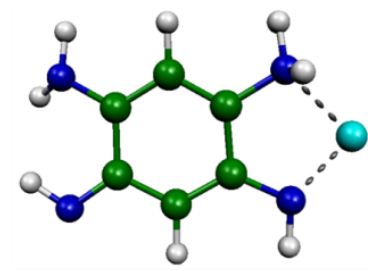

$m-\mathrm{DCD}(1.247 \mathrm{eV})$

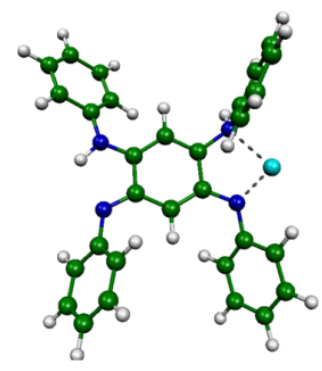

$m-\operatorname{AP}(0.724 \mathrm{eV})$

Figure S4 M06-2X/6-31+G(d) optimized structures of DCD and AP in the presence of a Cu atom. Energies relative to the ortho tautomer are given in parentheses.

\section{S2. Adsorption configurations of AP and AT}

\section{Azophenine, AP}

Upon deposition, we see a number of different molecular configurations on the $\mathrm{Cu}(110)$ surface. With four lobes indicating the presence of phenyl side groups, we can identify these molecules as AP (Figure S5). In addition to intact molecules, and a few buckled or deformed ones, we also see a minor degree of fragmentation evidenced by molecules possessing three lobes.

It is relatively straightforward to identify the species that exhibit a reversible switching property. As shown in Figure S5b, acquired over the same area as Figure S5a, but with a voltage above the activation threshold of $\pm 0.35 \mathrm{~V}$ for switching, we observe that some of the species appear fuzzy in appearance. This fuzziness is a direct consequence of the molecules switching at rates faster than the typical scan time over a single molecule. From many similar images, we conclude that fragmented species and deformed molecules do not display bistability. A necessary (but not sufficient) criterion for switching is that AP must be intact and adsorb in a planar configuration. The comparison of Figures S5a and S5b reveals that only two distinct adsorption configurations of intact, planar molecules exhibit switching (highlighted by green circles). These have all four phenyl lobes arranged in an undeformed square-like arrangement, and by defining the molecular axes running through two diagonally opposite phenyl lobes, these have their molecular axes either aligned as "addition" or "multiplication" signs in the field of view of Figures S5a and S5b. 

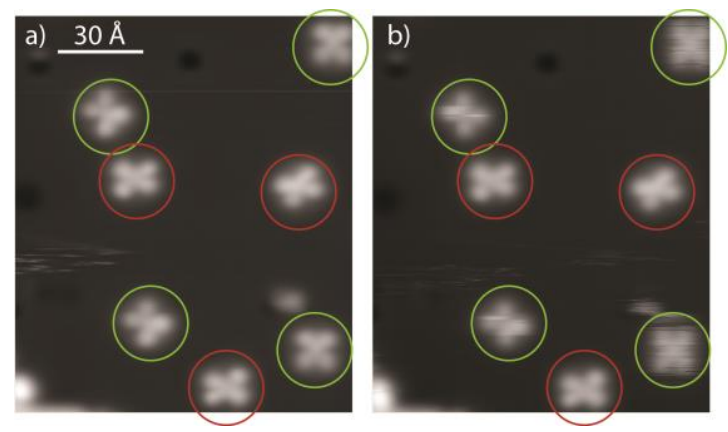

Figure S5 (a) STM image of assorted AP adsorption configurations ( $=1.40 \mathrm{nA}, \mathrm{V}=-0.18 \mathrm{~V})$. Green circles indicate molecules capable of switching; red circles indicate buckled molecules which do not switch. (b) STM of the same area but with a higher scanning bias showing the rapidly switching molecules highlighted in green $(\mathrm{I}=0.66 \mathrm{nA}, \mathrm{V}=-0.48 \mathrm{~V})$.

To deduce which of the described configurations adopts the energetically lowest structure, a simple annealing experiment was performed. The cleaned $\mathrm{Cu}(110)$ crystal was cooled to $100 \mathrm{~K}$ and AP was deposited to yield a low coverage as shown in Figure S6a. The prepared sample was then heated to $220 \mathrm{~K}$ followed by cooling to $78 \mathrm{~K}$. As seen in Figure S6b, the molecules have diffused over the surface to form small islands now composed of a single species: those in the shape of an addition sign. We therefore can conclude that these molecules have found the global minimum in potential energy and represent the ground state species adsorbed on the surface. This is the AP configuration which forms the bulk of the main study and details of its adsorption registry with respect to the underlying substrate can be found in Section S5.

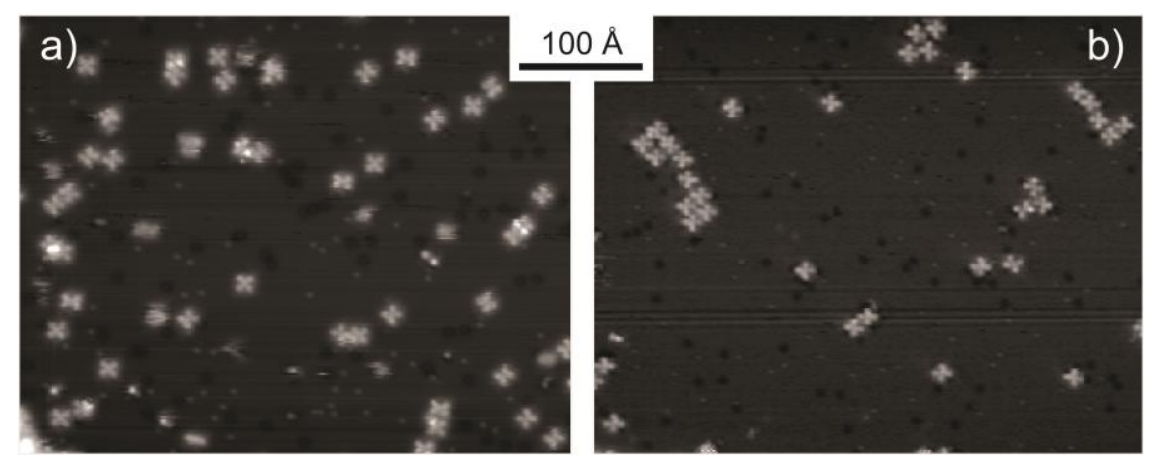

Figure S6 (a) STM image of adsorbed AP molecules displaying a number of different configurations $(I=0.62 n A$, $\mathrm{V}=-0.81 \mathrm{~V}, \mathrm{~T}=78 \mathrm{~K}$ ). (b) STM image after the sample was annealed to $220 \mathrm{~K}$. The AP molecules have adopted the lowest energy structure $(\mathrm{I}=0.69 \mathrm{nA}, \mathrm{V}=1.09 \mathrm{~V}, \mathrm{~T}=78 \mathrm{~K})$.

\section{Azotolyline, $A T$}

AT was deposited on $\mathrm{Cu}(110)$ following the same recipe as for AP. The extent of fragmentation in AT is significantly higher compared to AP (Figure S7): approximately 95\% for AT, compared to less than $5 \%$ for AP. We speculate that the intramolecular bonding in 
AT may be weakened by the presence of extra methyl groups in the para positions of the peripheral aromatic groups. The significant fragmentation of AT made it difficult to study all adsorption configurations. Therefore, to prove that the switching behavior was present, we focused on AT molecules with the same adsorption configuration with respect to the underlying substrate as AP.

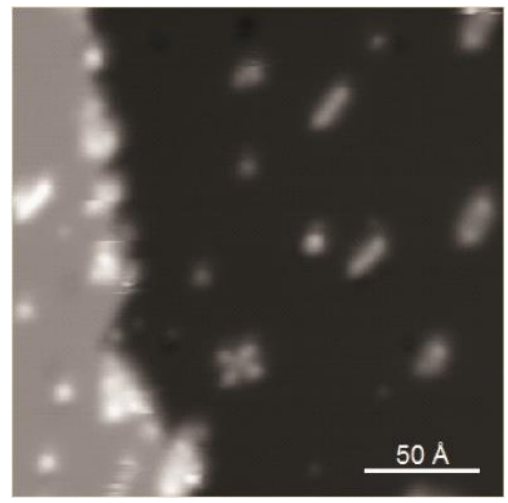

Figure S7 Typical STM image after deposition of AT on $\mathrm{Cu}(110)(\mathrm{I}=0.03 \mathrm{nA}, \mathrm{V}=-0.06 \mathrm{~V})$. The intact isolated molecule in the lower half of the image is accompanied by molecular fragments.

\section{S3. Observation of switching within molecular islands}

Proof that the observed switching of AP is not due to a physical $180^{\circ}$ rotation of the molecule is found in the observation of switching events in ordered arrays of molecules. As seen in Figure S8, the circled molecule shows the characteristic change in contrast of opposite lobes after a switch has taken place. The change was induced by scanning over the group of molecules with a bias voltage higher than the threshold for switching. Rotation of a molecule in this situation would be sterically hindered and hence can be ruled out. The mechanism of switching must therefore be due to an internal change in the configuration of the molecule (i.e. tautomerization).
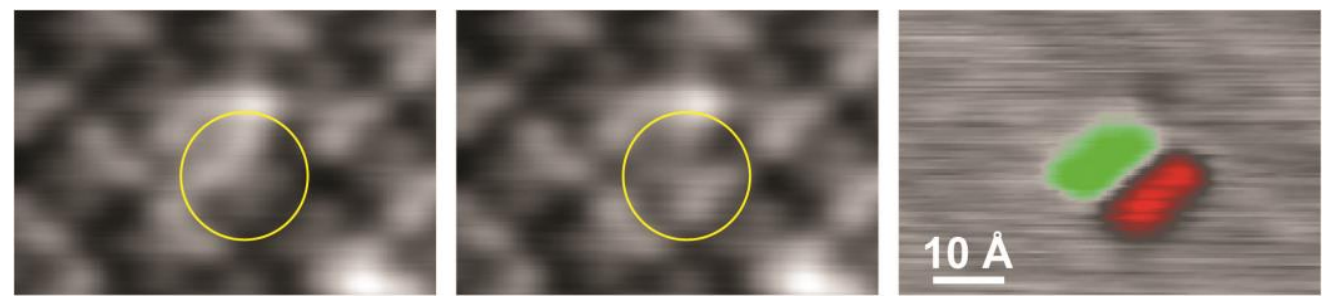

Figure S8 STM images before (left) and after (middle) observation of a switch of an AP molecule (circled) locked into a molecular island. ( $=0.63 \mathrm{nA}, \mathrm{V}=-0.15 \mathrm{~V}$ ). A difference image of the STM images (right) indicates the change in apparent height of the switched molecule. 


\section{S4. Details of periodic DFT calculations}

Periodic DFT calculations were carried out using the VASP program, ${ }^{5-7}$ using the PBE functional, ${ }^{8}$ a plane-wave basis set with an energy cut-off of $400 \mathrm{eV}$, PAW (Projector Augmented Wave $)^{9}$ treatment of core electrons and the VASP-supplied pseudopotentials for this method. ${ }^{10}$ Dipole correction ${ }^{11}$ was applied to the direction orthogonal to the surface, and the D2 empirical VdW correction ${ }^{12}$ was applied. The $\mathrm{Cu}(110)$ surface was represented as a slab of four atomic layers, with a rectangular unit cell of $17.82 \times 20.17 \AA$ ( 5 × 8 surface atoms, resulting in $160 \mathrm{Cu}$ atoms). During geometry optimization, the adsorbate as well as the uppermost two Cu layers were allowed to relax, while the lower two layers were frozen at the optimized bulk geometry. Simulated STM images were produced using the bSkan package. ${ }^{13,14}$

The optimized geometries for the four possible meta- and para-AP structures were computed and are displayed in Figure S9. The two equivalent meta-AP configurations observed experimentally have two possible reaction pathways (meta $\rightarrow$ para-1 $\rightarrow$ meta, and meta $\rightarrow$ para- $\rightarrow$ meta) as described in the main article.
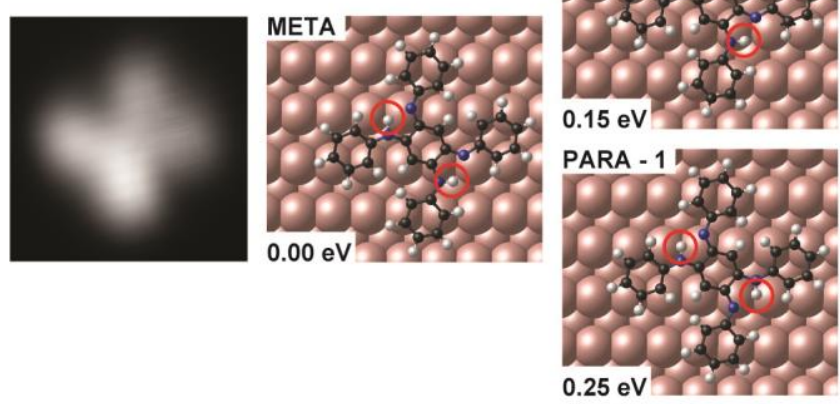

\section{Reaction Coordinate}

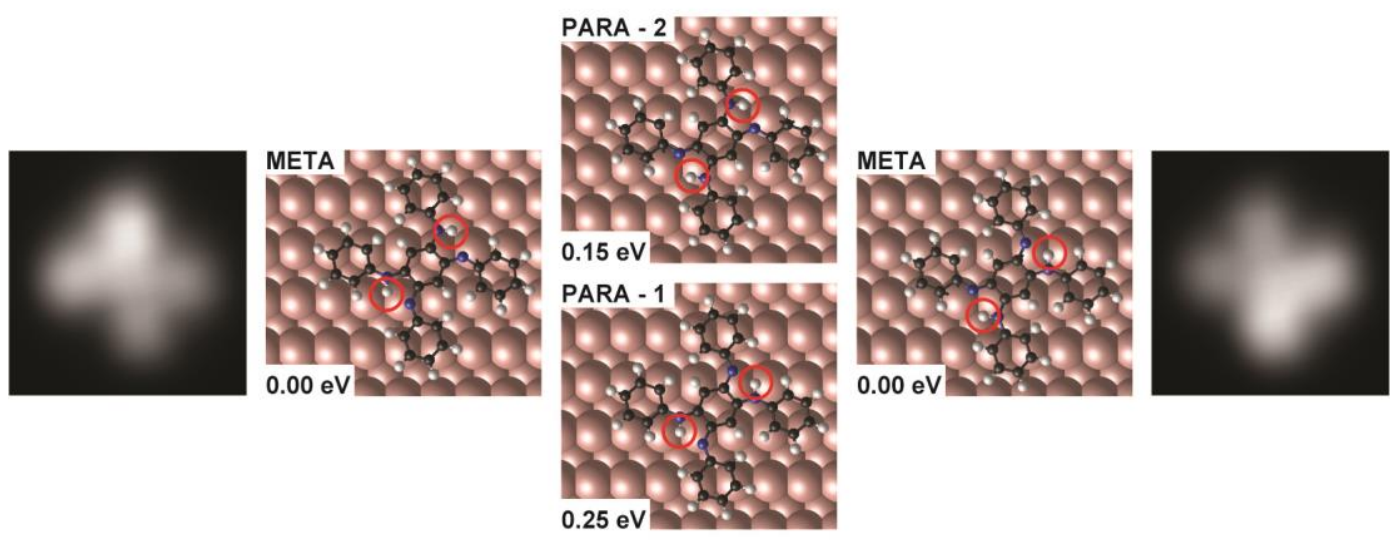

Figure S9 Scheme showing the four possible, energy equivalent meta-AP structures observed experimentally (as shown by accompanying STM images). Also shown are the four possible optimized para-AP structures, which act as reaction intermediates. 
Barrier heights were determined using the Nudged Elastic Band method. ${ }^{15}$ In an initial optimization, the meta and para structures were used as endpoints of the "band", and 60 optimization steps carried out, starting at eight geometries determined by linear interpolation using the "VASP TST Tools" package. ${ }^{16}$ The barrier was then refined by a further run using the climbing image NEB method between the geometries corresponding to the points left and right of the maximum in the pre-optimization, using the modified VASP routines from the Henkelmann group. ${ }^{17}$

The NEB calculation involving simultaneous transfer of both hydrogen atoms (i.e. concerted mechanism) for AP converges to a path with two maxima and an intermediate as shown in Figure S10. Upon inspection, the intermediate is identified as the para-2 state (see Figure 4 in main article), which indicates that a two-step process involving para-AP is favored over simultaneous transfer of both hydrogen atoms.

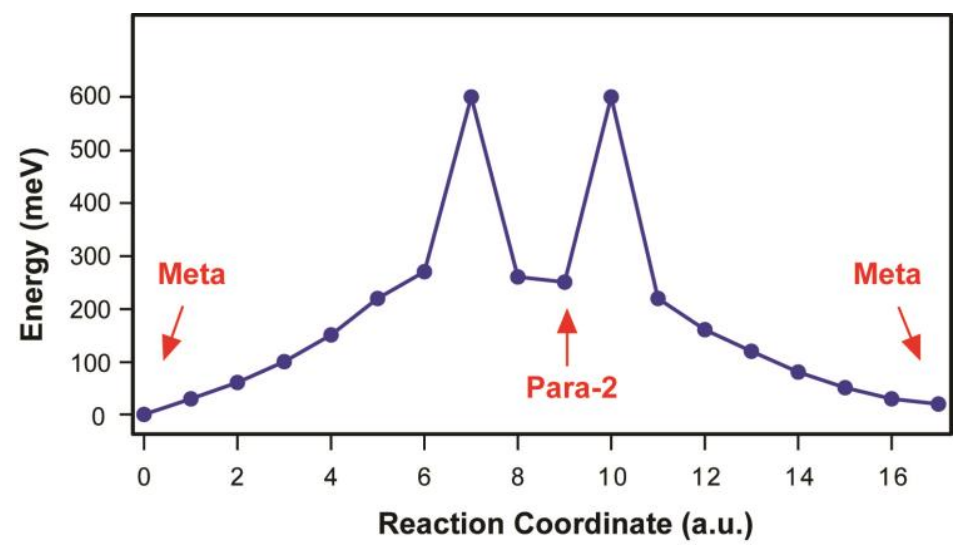

Figure S10 Converged reaction pathway for the concerted double proton meta-AP to meta-AP mechanism pointing to the existence of para intermediate, hence to a sequential single proton transfer mechanism.

\section{S5. Adsorption registry determination of AP}

In order to determine the precise adsorption registry of AP with respect to the $\mathrm{Cu}(110)$ lattice, a reconstructed surface, whose geometric structure is accurately known, ${ }^{18,19}$ was used due to difficulties in resolving the bare substrate atoms and the molecule simultaneously. Partial $\mathrm{Cu}(110)-\mathrm{O}(2 \times 1)$ was achieved by exposure of the surface to a low pressure of $\mathrm{O}_{2}$ and yielded stripes of reconstructed regions containing easily resolvable additional $\mathrm{Cu}$ atoms as shown in Figure S11a. After identical deposition parameters, AP was observed to preferentially adsorb on the bare $\mathrm{Cu}(110)$. From this it was unambiguously determined that the central ring of AP lies in the $\mathrm{Cu}(110)$ 4-fold coordination site, in agreement with our DFT calculations. In one direction, the lobes of the molecule are aligned 
along the [-110] direction and in the other direction the lobes lie along the [001] direction (Figure S11b and c).
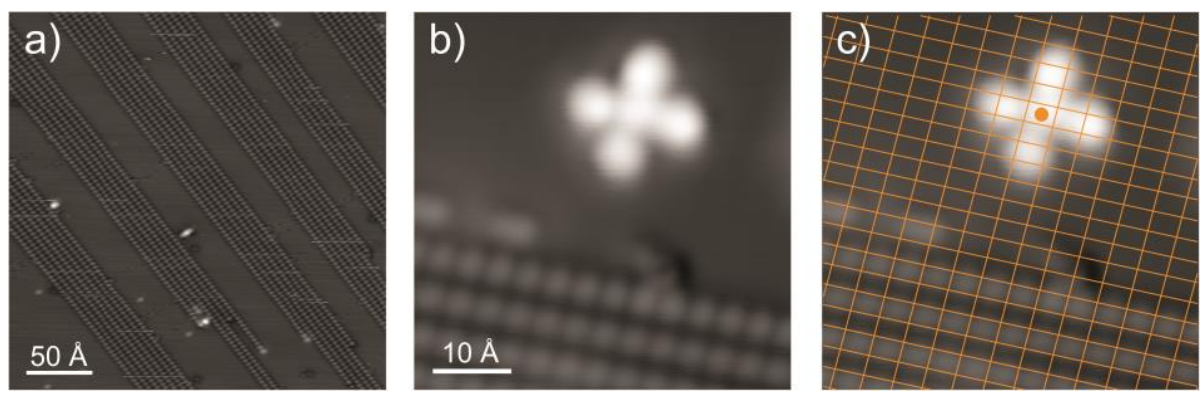

Figure S11 (a) Partially reconstructed $\mathrm{Cu}(110)-\mathrm{O}(2 \times 1)$ surface with rows of atomically resolved CuO on top of the bare $\mathrm{Cu}(110)$ substrate $(\mathrm{I}=1.00 \mathrm{nA}, \mathrm{V}=0.69 \mathrm{~V})$. (b) AP molecule adsorbed close to a reconstructed region with the $\mathrm{Cu}(110)$ lattice overlaid ( $=0.03 \mathrm{nA}, \mathrm{V}=0.251 \mathrm{~V})$. (c) The intersections in the overlaid grid coincide with the on-top positions of $\mathrm{Cu}(110)$. As indicated by a dot, the central ring of AP lies in the 4-fold coordination site.

\section{S6. Synthesis of azophenine (AP)}

To a solution of N,N'-diphenyl-p-quinone diimine $(0.5 \mathrm{~g}, 1.93 \mathrm{mmol})$ in chloroform $(25 \mathrm{ml})$ was added aniline $(0.4 \mathrm{ml}, 4.21 \mathrm{mmol}$ ) and a catalytic amount (a few crystals) of $\mathrm{p}$ toluenesulphonic acid. The solution was stirred overnight, and then $25 \mathrm{ml}$ of methanol was added to complete the precipitation of the dark red product. This was isolated by filtration, washed with methanol and dried to give $0.276 \mathrm{~g}(0.63 \mathrm{mmol})$ of product. Microanalysis for $\mathrm{C}_{30} \mathrm{H}_{24} \mathrm{~N}_{4}$ (AP): Calculated: C, 81.8; H, 5.5; N, 12.7 \%; Found C 80.8; H 5.4; N, 12.3 \%. The $\mathrm{N}, \mathrm{N}^{\prime}$-diphenyl-p-quinone diimine is made according to reference. ${ }^{20}$

\section{References}

1. Zhao, Y.; Truhlar, D. G. Theor. Chem. Acc. 2008, 120, 215-241.

2. Frisch, M. J.; Trucks, G. W.; Schlegel, H. B.; Scuseria, G. E.; Robb, M. A.; Cheeseman, J. R.; Scalmani, G.; Barone, V.; Mennucci, B.; Petersson, G. A.; Nakatsuji, H.; Caricato, M.; Li, X.; Hratchian, H. P.; Izmaylov, A. F.; Bloino, J.; Zheng, G.; Sonnenberg, J. L.; Hada, M.; Ehara, M.; Toyota, K.; Fukuda, R.; Hasegawa, J.; Ishida, M.; Nakajima, T.; Honda, Y.; Kitao, O.; Nakai, H.; Vreven, T.; Montgomery, J., J. A.; Peralta, J. E.; Ogliaro, F.; Bearpark, M.; Heyd, J. J.; Brothers, E.; Kudin, K. N.; Staroverov, V. N.; Kobayashi, R.; Normand, J.; Raghavachari, K.; Rendell, A.; Burant, J. C.; Iyengar, S. S.; Tomasi, J.; Cossi, M.; Rega, N.; Millam, N. J.; Klene, M.; Knox, J. E.; Cross, J. B.; Bakken, V.; Adamo, C.; Jaramillo, J.; Gomperts, R.; Stratmann, R. E.; Yazyev, O.; Austin, A. J.; Cammi, R.; Pomelli, C.; Ochterski, J. W.; Martin, R. L.; Morokuma, K.; Zakrzewski, V. G.; Voth, G. A.; Salvador, P.; Dannenberg, J. J.; Dapprich, S.; Daniels, A. D.; Farkas, Ö.; Foresman, J. B.; Ortiz, J. V.; Cioslowski, J.; Fox, D. J. Gaussian 09, Rev. A.02, Wallingford CT, 2009.

3. Holloway, M. K.; Reynolds, C. H.; Merz, K. M. J. Am. Chem. Soc. 1989, 111, 3466-3468.

4. Frantz S.; Rall J.; Hartenbach I.; Scheid T.; Zális S.; Kaim W. Chem. Eur. J. 2004, 10, 149-154. 
5. Kresse, G.; Hafner, J. Phys. Rev. B 1993, 47, 558-561.

6. Kresse, G.; Furthmüller, J. Comput. Mat. Sci. 1996, 6, 15-50.

7. Kresse, G.; Furthmüller, J. Phys. Rev. B 1996, 54, 11169-11186.

8. Perdew, J. P.; Burke, K.; Ernzerhof, M. Phys. Rev. Lett. 1996, 77, 3865-3868.

9. Blöchl, P. E. Phys. Rev. B 1994, 50, 17953-17979.

10. Kresse, G.; Joubert, D. Phys. Rev. B 1999, 59, 1758-1775.

11. Neugebauer, J.; Scheffler, M. Phys. Rev. B 1992, 46, 16067-16080.

12. Grimme, J. J. Comp. Chem. 2006, 27, 1787-1799.

13. Hofer, W. A.; Redinger, J. Surf. Sci. 2000, 447, 51-61.

14. Hofer, W. A. Prog. Surf. Sci. 2003, 72, 147-183.

15. Mills, G.; Jónsson, H.; Schenter, G. K. Surf. Sci. 1995, 324, 305-337.

16. Jónsson, H.; Mills, G.; Jacobsen, K. W. Classical and Quantum Dynamics in Condensed Phase Simulations Ch. 8 'Nudged Elastic Band Method for Finding Minimum Energy Paths of Transitions', Ed. B. J. Berne, G. Ciccotti and D. F. Coker (World Scientific, 1998).

17. Henkelman, G.; Uberuaga, B. P.; Jónsson, H. J. Chem. Phys. 2000, 113, 9901-9904.

18. Coulman, D. J.; Wintterlin, J.; Behm, R. J.; Ertl, G. Phys. Rev. Lett. 1990, 64, 1761-1764.

19. Jensen, F.; Besenbacher, F.; Laegsgaard, E.; Stensgaard, I. Phys. Rev. B 1990, 41, 1023310236.

20. Honzl, J.; Metalová, M. Tetrahedron 1969, 25, 3641-3652. 